ORNL/TM--11262

DE91 000522

Instrumentation and Controls Division

\title{
OPTIMIZING THE TEMPERATURE COMPENSATION OF AN ELECTRONIC PRESSURE MEASUREMENT SYSTEM
}

L. C. Maxey

T. V. Blalock

Date Published-August 1990

Prepared for the

David Taylor Research Center

under Interagency Agreement 40-1350-83

Prepared by the

OAK RIDGE NATIONAL LABORATORY

Oak Ridge, Tennessec 37831-6285

operated by

MARTIN MARIETTA ENERGY SYSTEMS, INC. for the

U.S. DEPARTMENT OF ENERGY

under Contract DE-AC(15-84OR21400) 


\section{CONTENTS}

Page

LIST OF FIGURES $\ldots \ldots \ldots \ldots \ldots \ldots \ldots \ldots \ldots \ldots \ldots \ldots$

ABSTRACT $\ldots \ldots \ldots \ldots \ldots \ldots \ldots \ldots \ldots \ldots \ldots \ldots \ldots \ldots \ldots \ldots \ldots \ldots$

1. INTRODUCTION ........................ 1

2. PRESSURE MEASUREMENT SYSTEM $\ldots \ldots \ldots \ldots \ldots \ldots \ldots \ldots \ldots$

2.1 Sources of Temperature Sensitivity $\ldots \ldots \ldots \ldots \ldots \ldots \ldots \ldots \ldots \ldots$

2.2 Temperature Sensitivity Characteristic Curve ................ 4

2.3 Transducer Temperature Compensation $\ldots \ldots \ldots \ldots \ldots \ldots \ldots \ldots \ldots \ldots \ldots$

2.4 Optimizing Sensitivity Compensation $\ldots \ldots \ldots \ldots \ldots \ldots \ldots \ldots \ldots \ldots \ldots \ldots$

3. EXPERIMENTAL RESULTS $\ldots \ldots \ldots \ldots \ldots \ldots \ldots \ldots \ldots \ldots \ldots \ldots \ldots$

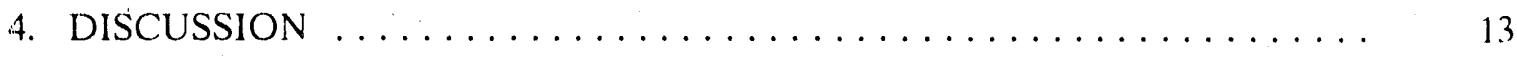

5. ACKNOWLEDGMENTS $\ldots \ldots \ldots \ldots \ldots \ldots \ldots \ldots \ldots \ldots \ldots \ldots \ldots$

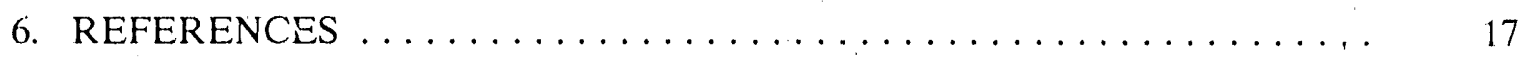

Appendix A. SENSITIVITY ANALYSIS ................. 19

$\begin{array}{ll}\text { Appendix B. } & \text { SENSITIVITY COMPENSATION RESISTANCE } \\ & \text { SELECTED BY SENSITIVITY ANALYSIS } \ldots \ldots \ldots \ldots \ldots \ldots\end{array}$

Appendix C. AVERAGE TEMPERATURE COEFFICIENTS . . . . . . . . 25 


\section{LIST OF FIGURES}

Figure

Pige

1. Typical sensor/electronics module pressure measurement

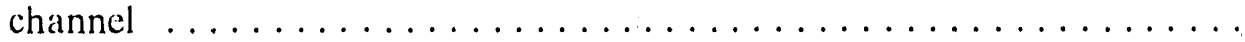

2. Schematic diagram showing locations of compensation resistors on

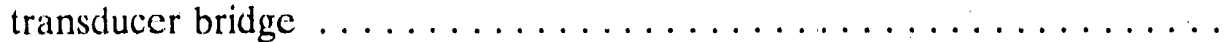

3. Calculated sensitivity curve optimized for -1 to $54^{\circ} \mathrm{C} \ldots \ldots \ldots \ldots \ldots$

4. Calculated sensitivity curve optimized for 0 to $25^{\circ} \mathrm{C} \ldots \ldots \ldots \ldots$

5. Change in sensitivity measured at 0 and $25^{\circ} \mathrm{C}$ using several values

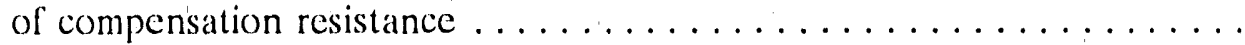

6. Change in measured pressure with temperature using empirically selected compensation resistance and resistance supplied with transducer . . . . . .

7. Change in measured pressure with temperature using calculated compensation resistance and resistance supplied with transducer . . . . . . .

B.1. Measurement system equivalent circuit $\ldots \ldots \ldots \ldots \ldots \ldots \ldots \ldots \ldots \ldots$

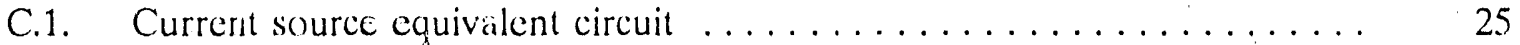




\begin{abstract}
In an effort to minimize temperature sensitivity the pressure measurement channels in the sensor/electronics modules of a high-resolution multiplexed pressure meastirement system were analyzed. The pressure sensor (a silicon diaphragm strain gage) was known to have two temperature-dependent parameters. Component testing revealed that the current source driving the pressure sensor was also temperature sensitive. Although the transducer manufacturer supplies empirically selected temperature compensation resistors with each transducer, it was determined that the temperature sensitivity compensation could be optimized for this application by changing one of these resistors. By modifying the value of the sensitivity compensation resistor to optimize performance in this application, the temperature sensitivity of the pressure measurement channels was reduced by more than $60 \%$.
\end{abstract}




\section{INTRODUCTION}

A pressure measurement system was developed under an interagency agreement with the David Taylor Research Center to measure three-dimensional flow vectors. The system consists of specialized multiport Pitot tubes coupled to high-resolution pressure measurement channels whose outputs are multiplexed into a data acquisition system. The pressure measurement channels are contained in several identical modules, with seven channels per module.

Accurate calculation of three-dimensional flow vectors from multiport Pitot tubes requires precise pressure measurement at each port. Variations in pressure sensitivity from channel to channel can occur if temperature gradients occur within the module. Although the module temperatures are to be monitored during operation, the effects of internal temperature gradients cannot be ignored. For this reason, the overall temperature sensitivity of the pressure measurement channels was optimized for the nominal operating temperature, taking into account all of the temperature-sensitive components in the measurement channels. 


\section{PRESSURE MEASUREMENT SYSTEM}

A typical pressure measurement channel consisting of a current source, pressure transducer, ${ }^{1}$ and instrumentation amplifier is illustrated in Fig. 1. The current source provides excitation for the transducer, and the output of the transducer is connected to the instrumentation amplifier. The voltage across the transducer bridge of one of the seven pressure measurement channels is monitored to provide an indication of the operating temperature of the module. Eight multiplexed data channels (seven for pressure and one for temperature) leave the module through an output driver amplifier. The typical operating temperature range of the sensor $/$ electronics module is 0 to $25^{\circ} \mathrm{C}$. Because pressure measurement channels are temperature dependent, the accuracy of the pressure data from a channel can be improved by knowing the temperature at which the data were taken. Pressure calibrations of the modules will be performed at three temperatures within the operating temperature range. In addition, the voltage from the data channel that monitors the voltage drop across one of the transducer bridges will be recorded at each temperature. In operation, the temperature data for the module and the pressure data from each sensor can be compared to the calibration data to accurately determine the pressure at each sensor.

Under uniform temperature conditions, the combination of temperature and pressure data from a module would allow for accurate pressure measurements at any temperature. Because of variations in the power dissipation in different parts of the module, however, temperature gradients are expected to occur. These gradients could cause the module temperature data to differ from the actual temperature of a given pressure measurement channel. For that reason it is desirable to minimize the iemperature sensitivity of the pressure measurement channels.

\subsection{SOURCES OF TEMPERATURE SENSITIVITY}

The temperature sensitivity of the pressure measurement channels is due to temperature-sensitive parameters associated with the pressure transducer itself and with the current source that drives the transducer. The temperature coefficients are expressed as fractional changes per degree Celsius.

The pressure transducer, which consists of four silicon resistor strain gages connected in a bridge, has a positive coefficient of resistance (TCR) and a negative coefficient of sensitivity or gage factor $(T C G)$. The transducer is designed so that the magnitude of $T C R$ is greater than the magnitude of $T C G$. This factor is a key element in the sensitivity temperature compensation because the value of $T C R$ can easily be reduced by the addition of a low-TCR parallel resistance. By proper selection of this resistance, the positive temperature characteristic and negative temperature characteristic can be foreed to cancel at one temperature, which is the compensation temperature.

The bridge current temperature coefficient is made up of a combination of effects. Beta changes in the transistors tend to increase current with temperature. In addition, the transducer current sources are all supplied by a single $-12-\mathrm{V}$ regulator which is temperature sensitive, tending to increase the bridge current with temperature. The output impedance of the current sources is relatively low $(60 \mathrm{k} \Omega)$ and has a negative 


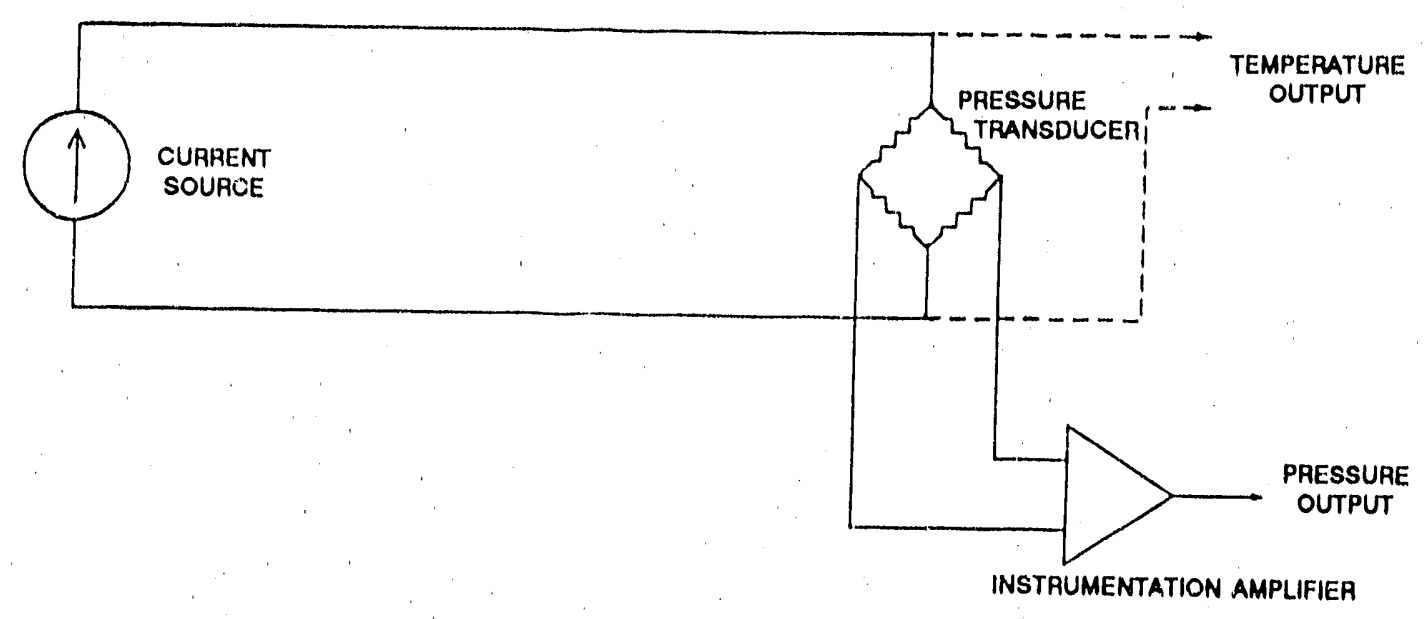

Fig. 1. Typical sensor/electronics module pressure measurement channel.

temperature coefficient. This parallel output impedance tends to reduce the bridge current as temperature increases.

By accounting for the temperature-dependent parameters affecting the current sources and using typical values of bridge resistance and current source output impedance, a single temperature coefficient for the transducer bridge current $(T C I)$ can be approximated.

\subsection{TEMPERATURE SENSITIVITY CHARACTERISTIC CURVE}

By evaluating the sensitivity expression for the silicon pressure transducers, ${ }^{2}$ the overall temperature sensitivity arising from the dominant temperature coefficients can be shown to have a characteristic shape. While the following example employs some approximations, the result is useful in understanding the problem of optimizing temperature compensation.

The pressure sensitivity of the pressure transducer can be expressed as

$$
S=G I R
$$

where

$$
\begin{aligned}
& S=\text { sensitivity }(\mathrm{mV} / \mathrm{psi}) \\
& G=\text { normalized sensitivity }(\mathrm{mv} / \mathrm{V} \text { of bridge voltage/psi) } \\
& I=\text { bridge current } \\
& R=\text { bridge resistance. }
\end{aligned}
$$

If the pressure sensitivity is expressed in terms of bridge voltage

$$
S=G V
$$


(where $V=I R$ ), the positive temperature coefficients of current and resistance can be combined into a single temperature coefficient of bridge voltage (TCV) where (for small variations in temperature)

$$
T C V=T C I+T C R
$$

The sensitivity at a temperature $T+\Delta T$ ( $T$ is the compensation temperature) can then be expressed (including temperature effects) as

$$
S=G[1+T C G(\Delta T)] V[1+T C V(\Delta T)]
$$

or

$$
S=G V\left[1+(T C G+T C V)(\Delta T)+(T C G)(T C V)(\Delta T)^{2}\right] .
$$

If $T C G$ and $T C V$ are opposite in sign and equal in magnitude, this expression reduces to

$$
S^{\prime}=G V\left[1+(T C G)(T C V)(\Delta T)^{2}\right] \text {. }
$$

The product of the two temperature cocflicients is always a negative constant, so the expression can be rewritten as

$$
S^{\prime}=V G\left[1-c(\Delta T)^{2}\right] \text {. }
$$

From the above expression for sensitivity, the characteristic shape of the sensitivity curve can be recognized as an inverted parabola centered about the compensation temperature. It should be noted that this curve is caused by the nonlinear dependence of $V$ and $G$ on temperature (a consequence of the fact that the temperature coefficients are defined as fractional changes per degree Celsius).

\subsection{TRANSDUCER TEMPERATURE COMPENSATION}

The pressure transducer as supplied by the manufacturer includes a set of precision metal film temperature compensation resistors. In Fig. 2, a transducer bridge is shown with five temperature compensation resistors. In practice, three of the five resistors are supplied with each transducer.

The temperature coefficients of the four resistors in the transducer bridge are not identical; therefore, either R1 or R.2 is used to reduce the effective temperature coefficient of one of the bridge resistors. As a result, the temperature coefficients of the two sides of the bridge will "track," reducing the drift in offset voltage with temperature. In addition, the offset voltage is recluced to $0.000 \mathrm{~V}$ (at $25^{\circ} \mathrm{C}$ ) by $\mathrm{R} 3$ and $\mathrm{R} 4$. Depending on the offset polarity. one of the two resistors is shorted and the other is used to balanee the bridge. 


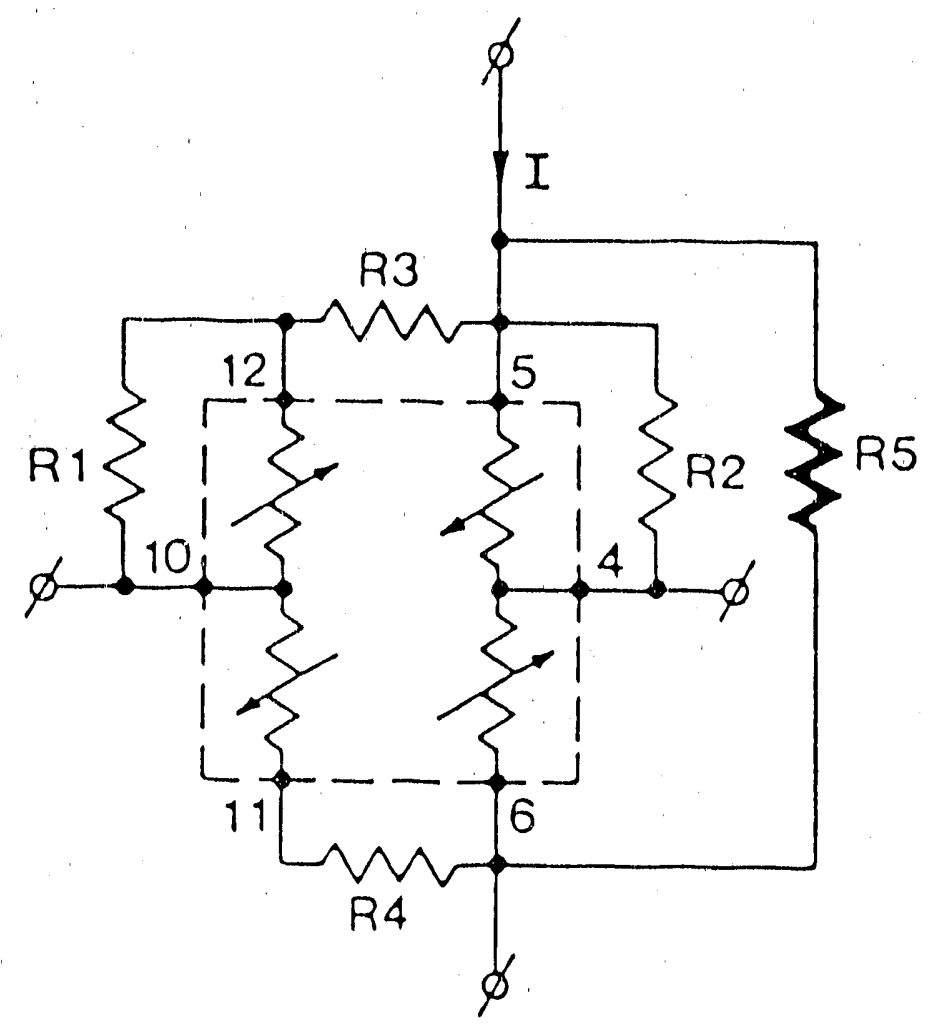

Fig. 2. Schematic diagram showing locations of compensation resistors on transducer bridge.

Sensitivity temperature compensation is achicved by reducing the magnitude of $T C V$ to the magnitude of $T C G$ to achieve the condition established in Eq. (6). The effective value of $T C V$ is reduced by adding a parallel resistor, R5, to the bridge. When R5 is included in the transducer network, the temperature coefficient $T C R$ applies to the parallel combination of the bridge and $\mathrm{R} 5$.

The value of R5 that is supplied with the transducer is selected to achieve the minimum variation in sensitivity over a temperature range from -1 to $54^{\circ} \mathrm{C}$. Because of the characteristic temperature compensation curve, the resulting compensu:ion temperature falls at about the midpoint of this range $\left(26.5^{\circ} \mathrm{C}\right)$, as illustrated in Fig. 3 .

For the purposes of the pressure measurement channels, there is no reason to modify the offset temperature compensation resistors (R1 and R2) or the offset adjustment resistors (R3 and R4), which are supplied by the transducer manufacturer. The sensitivity compensation resistor (R5), however, is not optimized for application in the present design of the pressure measurement channels.

The value of the sensitivity compensation resistor supplied with the transducer is selected to optimize performance over a broad temperature range when driven by a $1.5-\mathrm{mA}$ current source. The rurrent source must be either rernotely located at a stable temperature or insensitive to temperature effects. In this application, the temperature 


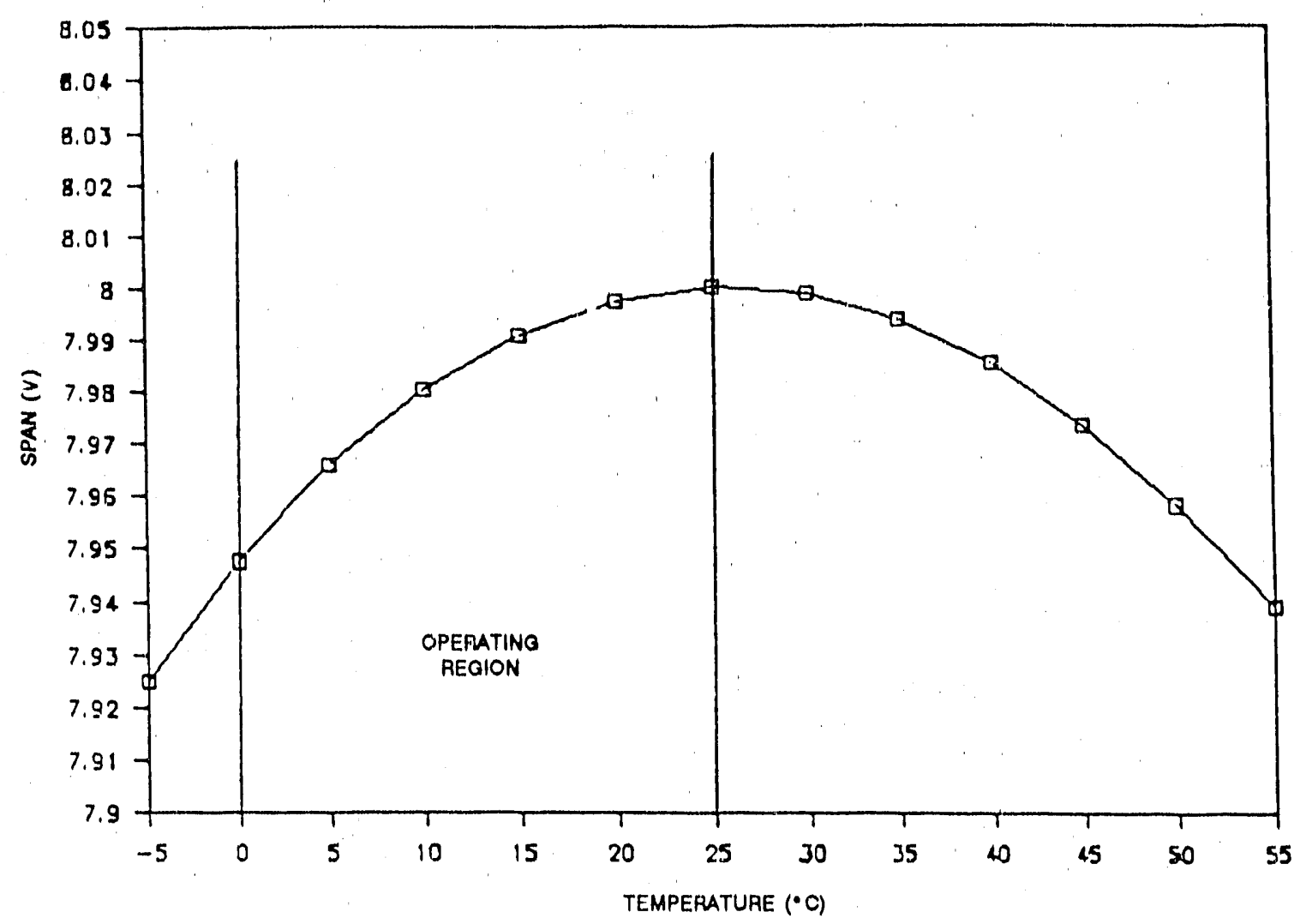

Fig. 3. Calculated sensitivity curve optimized for -1 to $54^{\circ} \mathrm{C}$.

range is narrower than the compensated range for the transducers. In addition, dense packaging requirements mandate the use of simple current sources that are temperature sensitive and are exposed to the same range of temperatures as the transducers. Both the operating range and the current source temperature coefficient can be taken into account to optimize the temperature sensitivity compensation for the pressure measurement channels.

\subsection{OPTIMIZING SENSITIVITY COMPENSATION}

Because of the par abolic nature of the sensitivity compensation curve, the total amount of variation in sensitivity due to temperature can be minimized by establishing the compensation temperature at the midpoint of the operating temperature range. This temperature is $-12.5^{\circ} \mathrm{C}$, as shown in Fig. 4.

The temperature coeflicient of bridge resistance is casily manipulated by the addition of parallel resistance. Temperature compensation is achieved when all the fractional temperature coefficients cancel. When R5 is added to the bridge network, it can be shown by sensitivity analysis (Appendix A) that the effective value of TCR becomes

$$
T C R=\left[R 5_{i}: R 5+R_{b}\right) \mid T C R_{b} .
$$


Since the value of $R_{b}$ varies directly with temperature, the value of TCR will vary inversely with temperature for a fixed value of R5. To establish the same value for $T C R$ at a lower temperature, Eq. (8) dictates that the value of $\mathrm{R} 5$ be decreased. It should be noted that the sensitivity of the transducer is reduced by lowering the value of R5. This effect is easily compensated by increasing the gain of the instrumentation amplifier.

The positive temperature coefficient associated with the current source tends to increase the compensation temperature. The low output impedance of the current source $(60 \mathrm{k} \Omega)$, however, reduces the effective value of $T C R$ by an amount that compensates for the increase and reduces the compensation temperature below the original value. The reduced value of $T C R$ does not shift the compensation temperature low enough for the measurement system requirements, however, so R5 must be reduced to achieve optimum compensation.

One method of optimizing R5 is by empirically testing the pressure measurement channel at the two extremes of the operating temperature range with a constant differential pressure applied to the transducer. By using several values of R5 and recording the output voltage at the two temperatures for each value, a resistance can be selected for which there should be no difference in the output voltage at the two temperature extremes. This selection is determined by plotting the difference in the output voltage at the tested temperatures vs the value of compensation resistance shown in Fig. 5. This selection process places the compensation temperature at the midpoint of the operating region indirectly by making the sensitivity equal at the temperature extremes.

The compensation temperature can be directly set if the temperature coefficients for the entire pressure measurement channel are known. This method accounts for all temperature-sensitive parameters in the electronics. Through sensitivity analysis (see Appendix B), it can be shown that the proper value of resistance to achieve compensation at a given temperature is given by

$$
R=-R_{b} \frac{1}{1+\frac{T C R_{b}}{T C G+T C I}+\frac{R_{b}}{R_{s}}\left[1+\frac{T C R_{s}}{T C G+T C I}\right]},
$$

where

$R_{b} \quad=$ bridge resistance at desired compensation temperature,

$R_{s} \quad=$ current source output impedance,

$T C R_{b}=$ temperature coefficient of bridge resistance,

$T C G=$ temperature coeflicient of transducer sensitivity,

$T C R_{s}=$ temperature coefficient of current source output impedance,

$T C I=$ temperature coefficient of current (internal to source).

The bridge resistance is the dominant temperature-sensitive term in this expression and provides the means for selecting a compensation temperature. If the value of $T C R_{b}$ is known, the bridge resistance can be measured at any temperature and $T C R_{b}$ applied to obtain the resistance at the desired compensation temperature. This value of bridge 


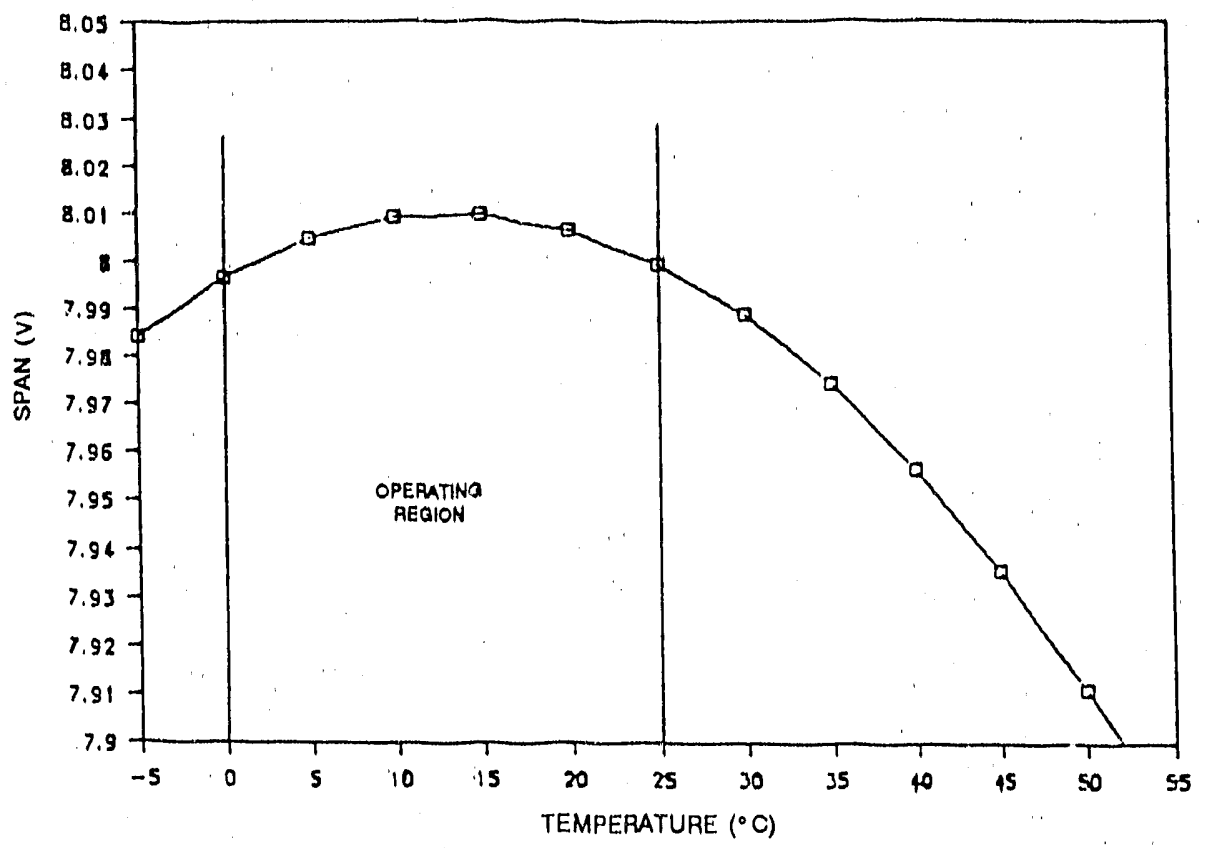

Fig. 4. Calculated sensitivity curve optimized for 0 to $25^{\circ} \mathrm{C}$.

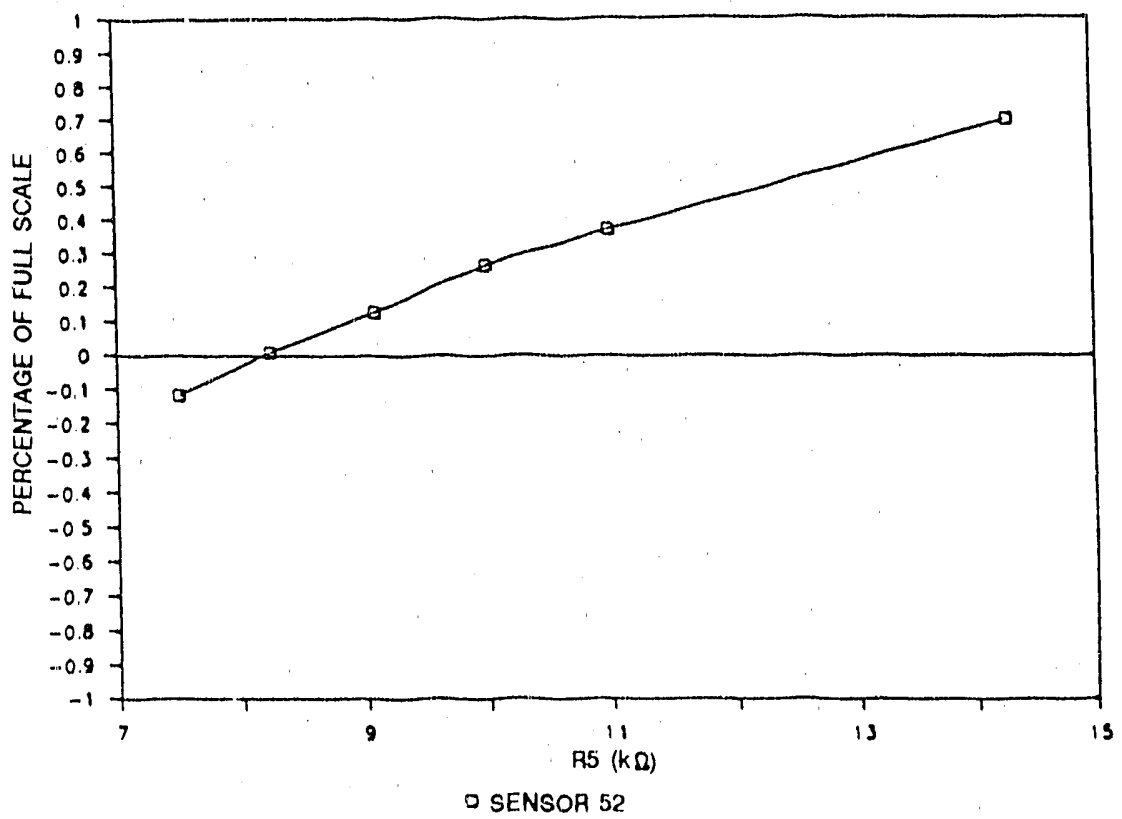

Fig. 5. Change in sensitivity measured at 0 and $25^{\circ} \mathrm{C}$ using several values of compensation resistance. 
resistance is then used in Eq. (9). Ideally, the value of each temperature-sensitive parameter would be determined for the desired compensation temperature prior to using Eq. (9).

Because of the large number of current sources present in this system, it is not practical to determine temperature coefficients for each one. The average temperature coefficients associated with the current sources (two different types exist in the present design) have been experimentally determined and are included in Appendix C.

Typical values for the sensor temperature coefficients $\left(T C R_{b}\right.$ and $\left.T C G\right)$ are not adequate for use in Eq. (9), because variations in these coefficients within the manufacturer's specified maximum and minimum values produce significant changes in the indicated value of compensation resistance. These coefficients must therefore be obtained from the manufacturer for each transducer. The value of bridge resistance and the temperature at which it was measured can also be obtained from the manufacturer with the coefficient data. These data are archived by the manufacturer but are not routinely distributed with the transducers. A small dearchiving fee is thus incurred in obtaining the data. 


\section{EXPERIMENTAL RESULTS}

The temperature sensitivity of two pressure measurement channels was tested by measuring the output voltage with a constant differential pressure at several temperatures in the operating range. One channel was tested by using the nearest available value of resistance to the value indicated by empirical testing, while the other used the nearest value to the resistance indicated by Eq. (9). In addition, each channel was tested by using the value of sensitivity-compensation resistance (R5) supplied by the manufacturer. This test permitted the amount of improvement in temperature sensitivity due to changing R5 to be determined for each case.

For one pressure measurement channel, the empirical test results shown in Fig. 5 were used to select the new value of R5. The resistor supplied by the manufacturer was $14.3 \mathrm{k} \Omega$ and the value indicated empirically was $\sim 8.1 \mathrm{k} \Omega$, a decrease of $43 \%$. An $8.25 \mathrm{k} \Omega$ resistor was selected from available stock. The results of the temperature tests are plotted in Fig. 6 (for clarity, the measurement channel output voltages have been converted to pressure). The total change in measured pressure over the range of temperatures tested was 0.083 psi when the original value of R5 was used and $0.030 \mathrm{psi}$ when the reduced value was used, which represents a $64 \%$ reduction in temperature sensitivity of the pressure measurement channel.

The temperature coefficients $T C R$ and $T C G$ were not available at the time of testing for specific transducers. To test the method of selecting R5 by use of Eq. (9), it was therefore necessary to start with the typical values of $T C R$ and $T C G$. The typical value for $T C R$ as provided by the manufacturer is $0.342 \% \% \mathrm{C}$ (minimum 0.306 , maximum 0.378 ). The typical value of $T C G$ is $-0.252 \% \% \mathrm{C}$ (minimum -0.234 , maximum -0.270 ). By evaluating the results of empirical tests with a computer model that incorporated all the temperature-dependent parameters, the typical values of $T C R$ and $T C G$ were manipulated slightly until agreement with empirical results was obtained. For the mathematical selection of R5, TCR and TCG were assigned values of 0.339 and -0.255 respectively.

A reduced value of $R 5$ was calculated by using Eq. (9) for a channel that had not been tested except for the measurement of bridge resistance. The calculated value for R5 was $7.95 \mathrm{k} \Omega$, a $34 \%$ reduction from the supplied value of $12.1 \mathrm{k} \Omega$. Again, the nearest available value at the time of testing was $8.25 \mathrm{k} \Omega$. The results of the temperature test are plotted in Fig. 7. The total change in indicated pressure over the range of temperatures tested was 0.083 psi when the original value of R5 was used and 0.029 psi when the recalculated value was used. This represents a $65 \%$ decrease in the temperature sensitivity of the pressure measurement channel. 


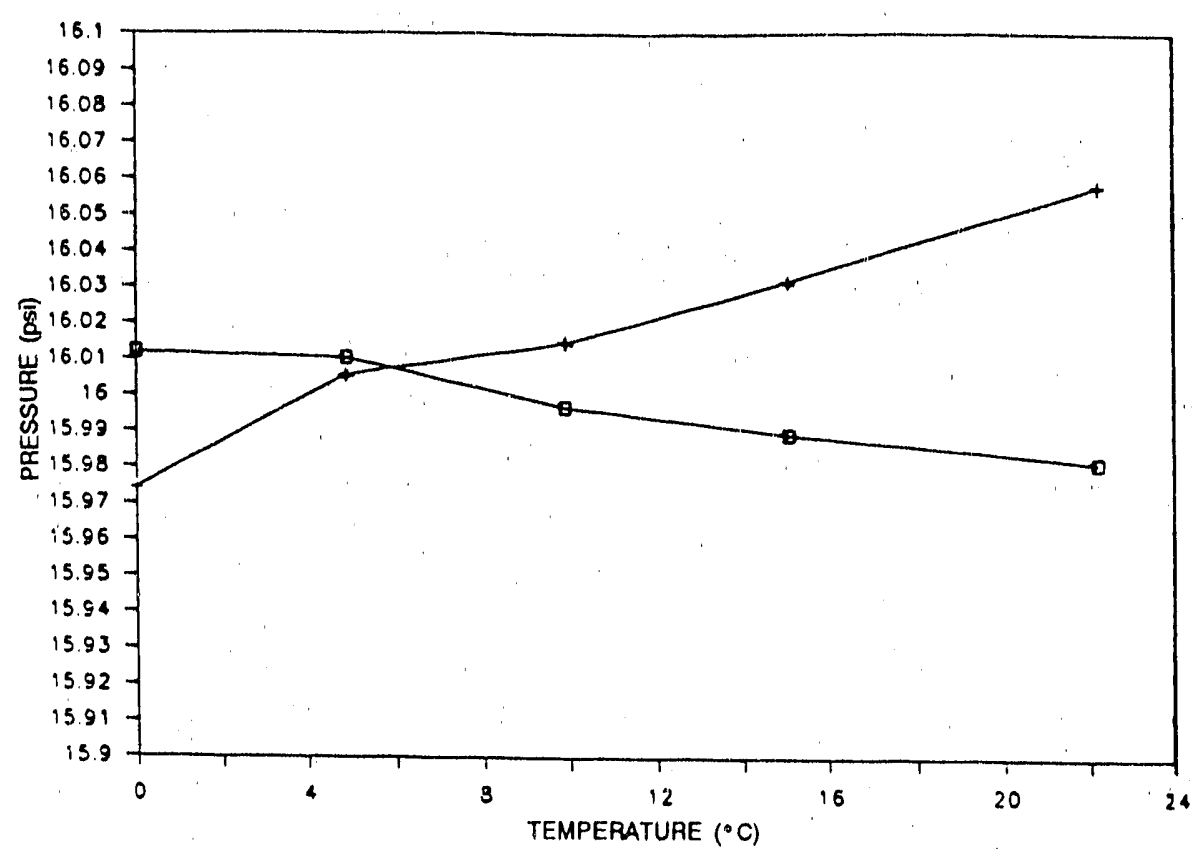

Fig. 6. Change in measured pressure with temperature using emperically selected compensation resistance (o) and resistance supplied with transducer $(+)$.

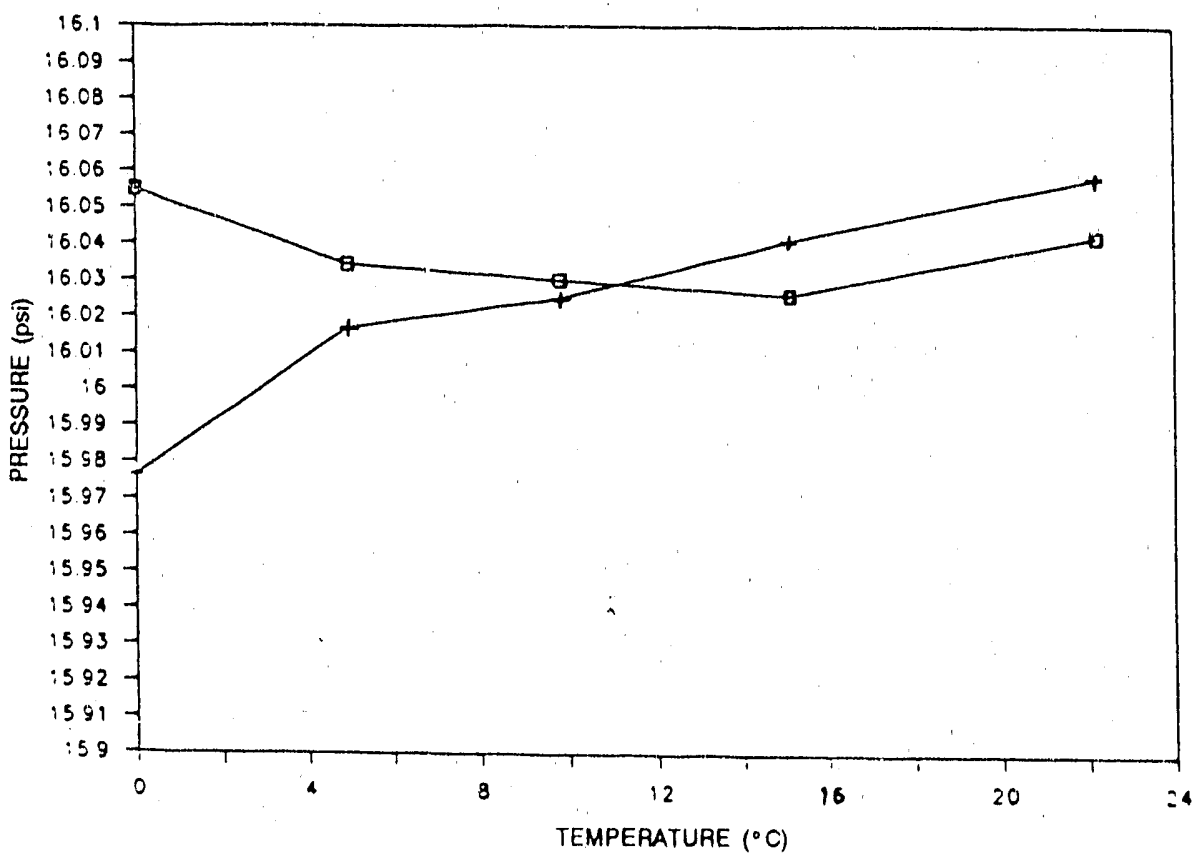

Fig. 7. Change in measured pressure with temperature using calculated compensation resistance $(0)$ and resistance supplied with transducer $(+)$. 


\section{DISCUSSION}

Some significant assumptions have been made in the analysis of temperature effects within the module:

- All temperature coefficients are assumed to remain constant over the range of operating temperatures.

- The self-heating effects due to current flow in the transducer have been neglected.

- Much of the sensitivity analysis is true only for "small" changes in temperature.

As optimum temperature compensation is approached, some of the second-order effects begin to become significant. As the results in Figs. 6 and 7 indicate, the temperature response using the reduced value of $\mathrm{R} 5$ is not an inverted parabola centered in the operating region, as is predicted by Eq. (7). This equation is predicated on the assumption that the three temperature-dependent parameters $(I, R$, and $G)$ can be reduced to two temperature-dependent parameters ( $V$ and $G$ ), which is true for only small changes in temperature.

The test results indicate that the temperature sensitivity of the pressure measurement channels can be reduced by more than $60 \%$ by optimizing the value of the span compensation resistor. In this application, the empirical method of selecting R5 is not feasible because of the large number of pressure measurement channels. The selection of R5 using Eq. (9) seemed to produce results comparable to those obtained by the empirical selection method, despite the fact that the exact values for TCR and TCG could not be obtained at the time of testing. When the actual values of $T C R$ and $T C G$ are obtained for each transducer, comparable or superior results are expected. 


\section{ACKNOWLEDGMENTS}

The authors express their gratitude to R. J. Fox, M. J. Roberts, and L. H. Thacker for many technical conversations pertinent to this research and for their editorial assistance. We also wish to express our appreciation to R. L. Shepard and R. W. Tucker, Jr., for their leadership and guidance. 


\section{REFERENCES}

1. F. Perrino, "Solid-State Pressure Sensors," Sensors 3(7), 44-49 (July 1986).

2. Temperature Compensation-IC Pressure Sensors, IC Sensors Application Note TN-002; March 1985. 


\section{Appendix A}

\section{SENSITIVITY ANALYSIS}

This appendix describes the determination of the temperature coefficient for the parallel combination of bridge resistance $\left(R_{b}\right)$ and any parallel resistance $(R)$ by sensitivity analysis.

The parallel combination of bridge resistance and any parallel resistance may be represented as

$$
\begin{gathered}
R_{p}=R_{b} \| R, \\
R_{p}=\frac{R_{b} R}{R_{b}+R},
\end{gathered}
$$

The differentinl may be expressed as

$$
\begin{gathered}
d R_{p}=\frac{\partial R_{p}}{\partial R_{b}} d R_{b}+\frac{\partial R_{p}}{\partial R} d R \\
d R_{p}=\frac{\left(R_{b}+R\right) R-R_{b} R}{\left(R_{b}+R\right)^{2}} d R_{b}+\frac{\left(R_{b}+R\right) R_{b}-R_{b} R}{\left(R_{b}+R\right)^{2}} d R .
\end{gathered}
$$

If the parallel resistance is relatively insensitive to temperature, the derivative with respect to temperature may be expressed as

$$
\frac{d R_{p}}{d T}=\frac{\left(R_{b}+R\right) R-R_{b} R}{\left(R_{b}+R\right)^{2}} \frac{d R_{b}}{d T}=\frac{R^{2}}{\left(R_{b}+R\right)^{2}} \frac{d R_{b}}{d T} .
$$

Rewriting this as the fractional change with temperature yields

$$
\frac{d R_{p}}{d T} \frac{1}{R_{p}}=\frac{R^{2}}{\left(R_{b}+R\right)^{2}} \frac{d R_{b}}{d T} \frac{1}{R_{p}}=\frac{R}{\left(R_{b}+R\right) R_{b}} \frac{d R_{b}}{d T}
$$

By rewriting this expression using temperature coefficients, we obtain

$$
T C R_{p}=\frac{R}{R_{b}+R} T C R_{b}
$$




\section{Appendix B \\ SENSITIVITY COMPENSATION RESISTANCE SELECTED BY SENSITIVITY ANALYSIS}

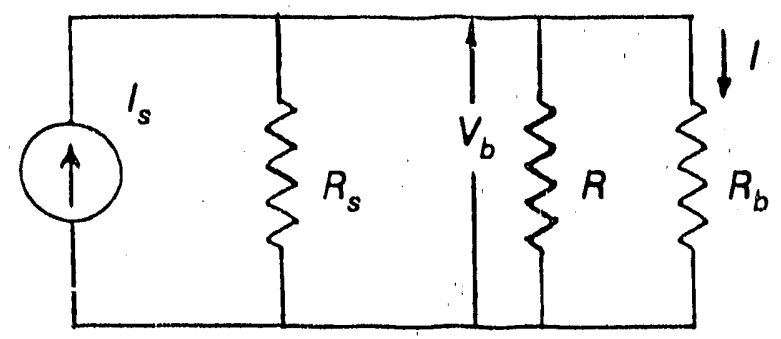

Fig. B.1. Measurement system cquivalent circuit.

$I_{s}=$ current source with temperature coefficient, $T C=T C I_{s}$;

$R_{s}=$ current source output impedance, $T C=T C R_{s}$;

$R_{b}=$ bridge resistance, $T C=T C R_{b}$

$I$ = current flowing into the transducer bridge;

$V_{b}=$ excitation voltage across the bridge;

$R=$ sensitivity compensation resistance.

The sensitivity of a pressure measurement channel may be expressed as

$$
S=G V_{b}(\mathrm{mV} / \mathrm{psi}) \text { or } S=G I R_{b}(\mathrm{mV} / \mathrm{psi}) .
$$

The quantity $G$ represents the normalized pressure sensitivity of the transducer $(\mathrm{mV} / \mathrm{psi} / \mathrm{mV}$ of applied bridge voltage). This quantity, also called the gage factor of the transducer, has a negative temperature coefficient, TCG.

To achieve temperature compensation, the fractional change in sensitivity with temperature must be zero, that is,

$$
\left(\frac{d S}{d T}\right) \frac{1}{S}=0
$$

Before differentiating the sensitivity expression, the quantity $I$ is found with respect to $I_{s}$ : 


$$
\begin{gathered}
I=I_{s} \frac{R_{s} \| R}{R_{s} \| R+R_{b}}=I \frac{\frac{R_{s} R}{R_{s}+R}}{\frac{R_{s} R}{R_{s}+R}+R_{b}}, \\
I=I \frac{R_{s} R}{R_{s} R+R_{b} R_{s}+R_{b} R} .
\end{gathered}
$$

The sensitivity expression may then be rewritten as

$$
S=G I_{s} \frac{R_{b} R_{s} R}{R_{s} R+R_{b} R_{s}+R_{b} R}=f\left(G, I_{s}, R_{b}, R_{s}, R\right)
$$

Differentiating with respect to $T$ yields

$$
\frac{d S}{d T}=\frac{\partial f}{\partial G} \frac{d G}{d T}+\frac{\partial f}{\partial I_{s}} \frac{d I_{s}}{d T}+\frac{\partial f}{\partial R_{b}} \frac{d R_{b}}{d T}+\frac{\partial f}{\partial R_{s}} \frac{d R_{s}}{d T}
$$

This expression assumes that the sensitivity compensation resistor is insensitive to temperaiure $(d R / d T=0)$.

The fractional change in sensitivity may be expressed as

$$
\left(\frac{d S}{d T}\right) \frac{1}{s}_{-}^{-}=\frac{G}{S} \frac{\partial f}{\partial G}\left(\frac{d G}{d T}\right) \frac{1}{G}+\frac{I_{s}}{S} \frac{\partial f}{\partial I_{s}}\left(\frac{d I_{s}}{d T}\right) \frac{1}{I_{s}}+\frac{R_{b}}{S} \frac{\partial f}{\partial R_{b}}\left(\frac{d R}{d T}\right) \frac{1}{R_{b}}+\frac{R_{s}}{S} \frac{\partial f}{R_{s}}\left(\frac{d R}{d T}\right) \frac{1}{R_{s}}
$$

or (rewriting the quantities in parentheses as temperature coefficients),

$$
T C S=\frac{G}{S} \frac{\partial f}{\partial G} T C G+\frac{I_{s}}{S} \frac{\partial f}{\partial I_{s}} T C I_{s}+\frac{R_{b}}{S} \frac{\partial f}{\partial R_{b}} T C R_{b}+\frac{R_{s}}{S} \frac{\partial f}{\partial R_{s}} T C R_{s} .
$$

Note that the multipliers of the temperature coefficients are "sensitivity functions." The sensitivity functions may be found from Eq. (B.4):

$$
\begin{gathered}
\frac{G}{S} \frac{\partial f}{\partial G}=\frac{G}{S} \frac{S}{G}=1 ; \\
\frac{I_{s}}{S} \frac{\partial f}{\partial I_{s}}=\frac{I_{s}}{S} \frac{S}{I_{s}}=1 ; \\
\frac{R_{b}}{S} \frac{\partial f}{\partial R_{b}}=\frac{R_{b}}{S} \frac{\frac{\left(R_{s} R+R_{b} R_{s}+R_{b} R\right)\left(R_{s} R\right)-\left(R_{b} R_{s} R\right)\left(R_{s}+R\right)}{\left(R_{s} R+R_{b} R_{s}+R_{b} R\right)^{2}} \quad G I_{s},}{,} \\
=\frac{R_{b}}{S} \frac{R_{s} R}{\left(R_{s} R+R_{b} R_{s}+R_{b} R\right)}-\frac{R_{b} R\left(R_{s}+R\right)}{\left(R_{s} R+R_{b} R_{s}+R_{b} R\right)^{2}} \quad G I_{s},
\end{gathered}
$$




$$
\begin{aligned}
& =\frac{R_{b}}{S} \frac{R_{s} R}{R_{s} R+R_{b} R_{s}+R_{b} R}-\frac{R_{b} R_{s} R\left(R_{s}+R\right)}{\left(R_{s} R+R_{b} R_{s}+R_{b} R\right)^{2}} S \frac{R_{s} R+K_{b} R_{s}+R}{R_{b} R_{s} R} \\
& =1-\frac{\left(R_{s}+R\right) R_{b}}{R_{s} R+R_{b} R_{s}+R_{b} R} .
\end{aligned}
$$

Similarly,

$$
\frac{R_{s}}{S} \frac{\partial f}{\partial R_{s}}=1-\frac{\left(R_{h}+R\right) R_{s}}{R_{s} R+R_{b} R_{s}+R_{b} R}
$$

Substituting for the sensitivity functions in Eq. (B.6), the sensitivity temperature coefficient may be expressed as

$$
T C S=T C G+T C I_{s}+T C R_{b} 1-\frac{\left(R_{s}+R\right) R_{b}}{R_{s} R+R_{b} R_{s}+R_{b} R}+T C R_{s} 1-\frac{\left(R_{b}+R\right) R_{s}}{R_{s} R+R_{b} R_{s}+R_{b} R} .
$$

To achieve temperature compensation, TCS must be set equal to zero; therefore,

$$
\begin{gathered}
T C G+T C I_{s}+T C R_{b}+T C R_{s}-\frac{T C R_{b}\left(R_{s}+R\right) R_{b}+T C R_{s}\left(R_{b}+R\right) R_{s}}{R_{s} R+R_{b} R_{s}+R_{b} R}=0 \\
\left(T C G+T C I_{s}+T C R_{b}+T C R_{s}\right)\left[R_{b}\left(R_{b}+R_{s}\right)+R_{b} R_{s}\right] \\
=R\left[R_{b}\left(T C R_{b}\right)+R_{s}\left(T C R_{s}\right)\right]+R_{b} R_{s}\left(T C R_{b}+T C R_{s}\right) \\
R\left[\left(R_{b}+R_{s}\right)\left(T C G+T C I_{s}+T C R_{b}+T C R_{s}\right)-R_{b}\left(T C R_{b}\right)-R_{s}\left(T C R_{s}\right)\right] \\
=R_{b} R_{s}\left[\left(T C R_{b}\right)+\left(T C R_{s}\right)-\left(T C G+T C I_{s}+T C R_{b}+T C R_{s}\right)\right. \\
R\left[R_{b}\left(T C G+T C I_{s}+T C R_{s}\right)+R_{s}\left(T C G+T C I_{s}+T C R_{b}\right)\right]=-R_{b} R_{s}\left(T C I_{s}+T C G\right) \\
R\left[\left(T C G+T C I_{s}\right)\left(R_{b}+R_{s}\right)+R_{b}\left(T C R_{s}\right)+R_{s}\left(T C R_{b}\right)\right]=-R_{b} R_{s}\left(T C I_{s}+T C G\right)
\end{gathered}
$$

An expression for the sensitivity compensation resistance may be written as

$$
\begin{gathered}
R=-R_{b} \frac{R_{s}\left(T C I_{s}+T C G\right)}{\left(T C G+T C I_{s}\right)\left(R_{b}+R_{s}\right)+R_{b}\left(T C R_{s}\right)+R_{s}\left(T C R_{b}\right)}, \\
R=-R_{b} \frac{1}{1+\frac{R_{b}}{R_{s}}+\frac{R_{b}\left(T C R_{s}\right)}{R_{s}\left(T C I_{s}+T C \bar{G}\right)}+\frac{T C R_{b}}{T C I_{s}+T C G}}, \\
R=-R_{b} \frac{1}{1+\frac{T C R_{b}}{T C I_{s}+T C G}+\frac{R_{b}}{R_{s}}\left[1+\frac{T C R_{s}}{T C G+T C I_{s}}\right]} .
\end{gathered}
$$


Appendix C

AVERAGE TEMPERATURE COEFFICIENTS

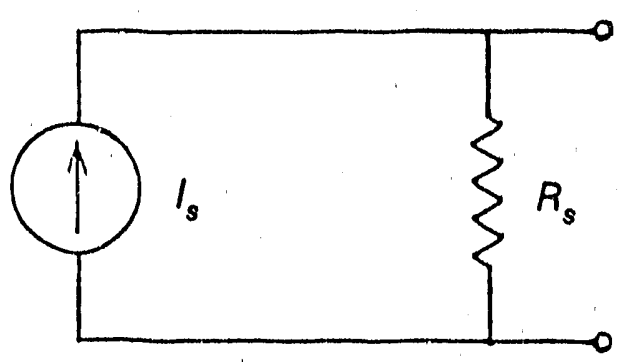

Fig. C.1. Current source equivalent circuit.

The average temperature coefficients for the two types of current sources used in the sensor/electronics modules have heen empirically determined:

Current source for channel 1

(MAT-(02)

$\begin{aligned} T C l_{s} & =0.037 \% /{ }^{\circ} \mathrm{C} \\ T C R_{s} & =-0.173 \% /{ }^{\circ} \mathrm{C} \\ \text { Average } R_{s} & =60.4 \mathrm{~K} @ 20^{\circ} \mathrm{C} \\ & =62.5 \mathrm{~K} @ 00^{\circ} \mathrm{C}\end{aligned}$

Current source for channels 2 through 7

(MAT-04)

$\begin{aligned} T C I_{s} & =0.057 \% /{ }^{\circ} \mathrm{C} \\ T C R_{s} & =-0.21 \% /{ }^{\circ} \mathrm{C} \\ \text { Average } R_{s} & =60.3 \mathrm{~K}(1) 25^{\circ} \mathrm{C} \\ & =63.4 \mathrm{~K}(u)\left(0^{\circ} \mathrm{C}\right.\end{aligned}$



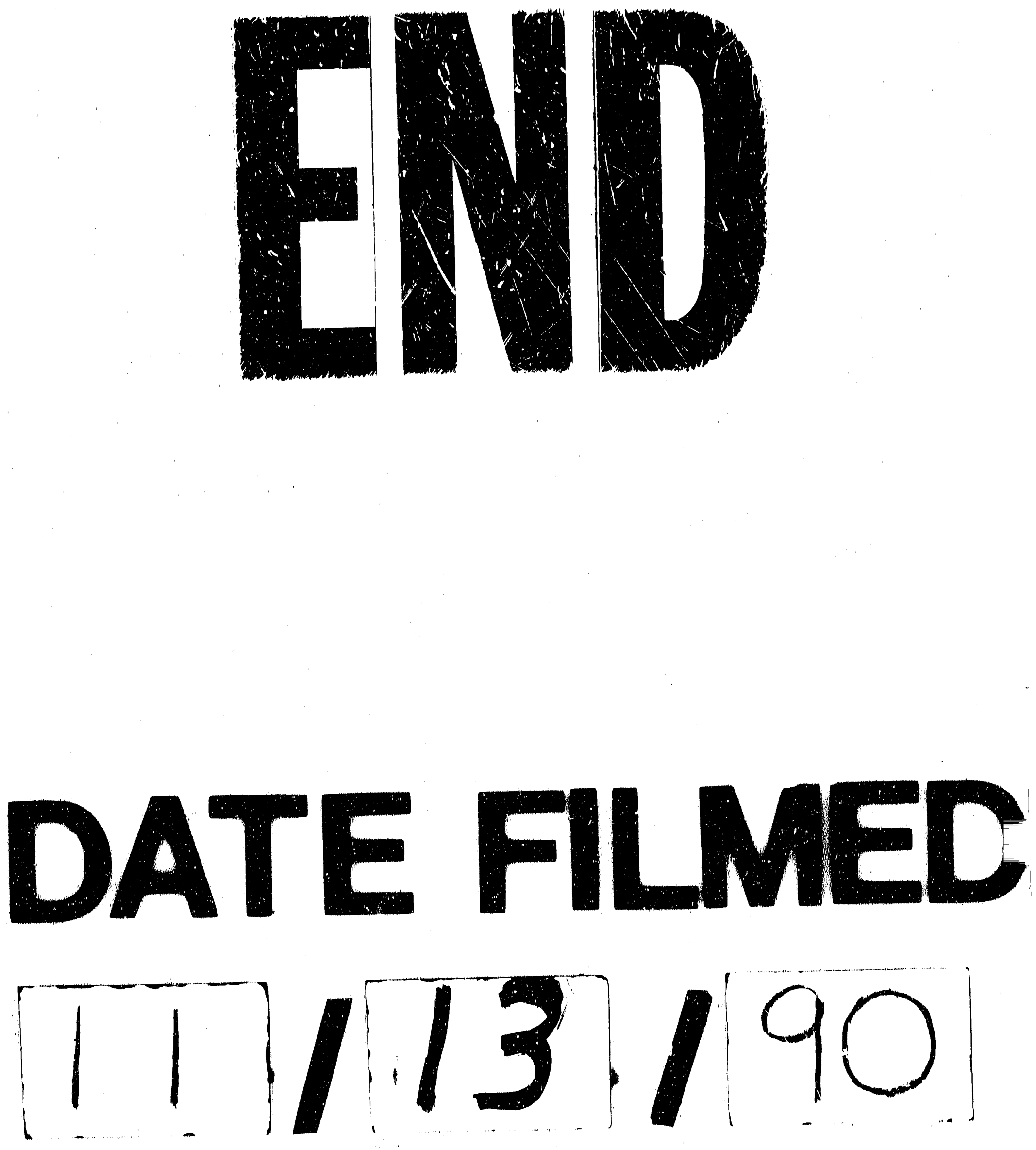
